\title{
Overview of the CMD-3 recent results at $e^{+} e^{-}$collider VEPP-2000
}

G. V. Fedotovich ${ }^{1,2, \star}$, R. R. Akhmetshin ${ }^{1}$, A. V. Anisenkov ${ }^{1,2}$, V. M. Aulchenko ${ }^{1,2}$, V. S. Banzarov ${ }^{1}$, N. S. Bashtovoy ${ }^{1}$, D. E. Berkaev ${ }^{1,2}$, A. V. Bragin ${ }^{1}$, S. I. Eidelman ${ }^{1,2}$, D. A. Epifanov ${ }^{1,5}$, L. B. Epshteyn 1,3 , A. L. Erofeev ${ }^{1,2}$, S. E. Gayazov ${ }^{1,2}$, A. A. Grebenuk ${ }^{1,2}$, D. N. Grigoriev ${ }^{1,2,3}$, E. M. Gromov ${ }^{1}$, F. V. Ignatov 1 , V. L. Ivanov ${ }^{1,2}$, S. V. Karpov1 ${ }^{1}$, V. F. Kazanin ${ }^{1,2}$, B. I. Khazin ${ }^{1,2}$, A. N. Kirpotin ${ }^{1}$, I. A. Koop ${ }^{1,2}$, O. A. Kovalenko ${ }^{1,2}$, A. N. Kozyrev ${ }^{1}$, E. A. Kozyrev ${ }^{1,2}$, P. P. Krokovny ${ }^{1,2}$, A. E. Kuzmenko ${ }^{1,3}$, A. S. Kuzmin ${ }^{1}$, I. B. Logashenko ${ }^{1,2}$, P. A. Lukin ${ }^{1,2}$, A. P. Lysenko ${ }^{1}$, K. Yu. Mikhailov ${ }^{1,2}$, V. S. Okhapkin ${ }^{1}$, Yu. N. Pestov ${ }^{1}$, E. A. Perevedentsev1,2, A. S. Popov ${ }^{1,2}$, G. P. Razuvaev ${ }^{1,2}$, Yu. A. Rogovsky ${ }^{1}$, A. L. Romanov¹, A. A. Ruban ${ }^{1}$, N. M. Ryskulov ${ }^{1}$, A. E. Ryzhenenkov ${ }^{1,2}$, V. E. Shebalin ${ }^{1,2}$, D. N. Shemyakin ${ }^{1,2}$, B. A. Shwartz ${ }^{1,2}$, D. B. Shwartz ${ }^{1,2}$, A. L. Sibidanov ${ }^{4}$, P. Yu. Shatunov ${ }^{1}$, Yu. M. Shatunov ${ }^{1}$, E. P. Solodov ${ }^{1,2}$, V. M. Titov ${ }^{1}$, A. A. Talyshever, ${ }^{1,2}$ A. I. Vorobiov' ${ }^{1}$, Yu. V. Yudin ${ }^{1}$, Yu. M. Zharinov' ${ }^{1}$

${ }^{1}$ Budker Institute of Nuclear Physics, SB RAS, Novosibirsk, 630090, Russia

${ }^{2}$ Novosibirsk State University, Novosibirsk, 630090, Russia

${ }^{3}$ Novosibirsk State Technical University, Novosibirsk, 630092, Russia

${ }^{4}$ University of Sydney, School of Physics, Falkiner High Energy Physics, NSW 2006, Sydney, Australia

${ }^{5}$ University of Tokyo, Department of Physics, 7-3-1 Hongo Bunkyo-ku Tokyo, 113-0033, Japan

\begin{abstract}
Since December 2010, the CMD-3 detector has collected data at the electronpositron collider VEPP-2000. The sample of the accumulated data corresponds to about $60 \mathrm{pb}^{-1}$ of integrated luminosity in the c.m. energy from 0.32 up to $2 \mathrm{GeV}$. Preliminary results of the analysis of various processes $e^{+} e^{-}$annihilation to hadrons are presented. It is shown the processes with multihadron events have several intermediate states which must be taken into account to correctly describe the angular and invariant mass distributions as well as cross section dependence versus energy.
\end{abstract}

\section{Introduction}

The electron-positron collider VEPP-2000 [1] operates at the Budker Institute of Nuclear Physics since December 2010. The collider is designed to provide luminosity up to $10^{32} \mathrm{~cm}^{-2} \mathrm{~s}^{-1}$ at the maximum center-of-mass energy $\sqrt{s}=2 \mathrm{GeV}$. The current integrated luminosity accumulated by the CMD-3 is about $60 \mathrm{pb}^{-1}$. One of the main physical task is to measure the hadronic cross sections in the wide energy range, and searches for the new vector and scalar mesons and as well as for the exotic hadrons.

The precision data of the hadronic cross sections are required for various applications, in particular, to evaluate the anomalous magnetic moment (AMM) of muon, $a_{\mu}=(g-2)_{\mu} / 2$. The VEPP-2000

^e-mail: fedotovich@inp.nsk.su 
energy range gives the major hadronic contribution to AMM ( $a_{\mu}^{\text {had }} \sim 92 \%$ ) both to the hadronic vacuum polarization itself and to its uncertainty [2].

The precision measurement of luminosity is a key ingredient of many experiments which study the hadronic cross sections at $e^{+} e^{-}$colliders. So far it is very important to have several QED processes such as $e^{+} e^{-} \rightarrow e^{+} e^{-}, \mu^{+} \mu^{-}, \gamma \gamma$ to have the cross check as it was done by CLEO [3].

The general purpose detector CMD-3 has been described in detail elsewhere [4]. Its tracking system consists of a cylindrical drift chamber (DC) and double-layer multiwire proportional Z-chamber, both also used for a trigger, and both inside a thin $\left(0.2 \mathrm{X}_{0}\right)$ superconducting solenoid with a field of $1.3 \mathrm{~T}$. The liquid xenon ( $\mathrm{LXe}$ ) barrel calorimeter with $5.4 \mathrm{X}_{0}$ thickness has fine electrode structure, providing 1-2 $\mathrm{mm}$ spatial resolution, and shares the cryostat vacuum volume with the superconducting solenoid. The barrel CsI crystal calorimeter [5] with thickness of $8.1 \mathrm{X}_{0}$ is placed outside the LXe calorimeter, and the end-cap BGO calorimeter with a thickness of $13.4 \mathrm{X}_{0}$ is placed inside the solenoid. The luminosity is measured using events of Bhabha scattering and $\gamma \gamma$ at large angles with about $1 \%$ accuracy.

The beam energy has been monitored by measuring the current in the dipole magnets of the main ring, and for a few energy points by using the Back-Scattering-Laser-Light system [6]. Using measured average momentum from Bhabha events, and average momentum of proton-antiproton pairs from the $e^{+} e^{-} \rightarrow p \bar{p}$ process, we determine $\mathrm{E}_{c} m$ for each energy point with about $1 \mathrm{MeV}$ accuracy.

To study a detector responce and to obtain a registration efficiency, we have developed a Monte Carlo (MC) simulation of our detector based on GEANT4 package, and all simulated events pass all our reconstruction and selection procedures. The MC simulation includes photon radiation by initial electron or positron, calculated according to Ref. [7].

\section{Luminosity measurement}

The energy range from 1 to $2 \mathrm{GeV}$ was scanned upward and downward with a step of $50 \mathrm{MeV}$. At each energy point the integrated luminosity of about $500 \mathrm{nb}^{-1}$ was accumulated. In the case of scanning downward, the energy points have been shifted by $25 \mathrm{MeV}$ with respect to the previous case. The experiments were performed from January to June 2011 and, similarly, in 2012.

Two types the «CHARGED» and the «NEUTRAL» first level triggers were used while data have been taking. A combinations of the signals from DC cells and ZC sectors, which roughly reproduce "track", start a special processor «TRACKFINDER» (TF). The combinations of signals from calorimeters with different energy thresholds actuated the «CLUSTERFINDER» $(\mathrm{CF})$ processor. A positive decision of either processor allowed the recording of current event onto a hard disk with capacity about 2 TB. In the course of data accumulation, the mean trigger frequency ranged in $\sim 200 \div$ $400 \mathrm{~Hz}$. The sample of collinear Bhabha events $e^{+} e^{-}$were selected for luminosity determination.

The process $e^{+} e^{-} \rightarrow \gamma \gamma$ was also used, since it has essential advantages [8] with respect to the first one. It is free of radiation of the final state particles and its Coulomb interaction, the corresponding Feynman graphs do not contain photon propagators affected by the vacuum polarization effects. These advantages are the main motivation to exploit this process as an independent tool for luminosity.

The collected integrated luminosity above the $\phi$ mass is about $34.5 p b^{-1}, 8.3$ and $8.4 p b^{-1}$ at the $\omega$ and $\phi$ resonances, respectively, and $9.4 \mathrm{pb}^{-1}$ from a scan below the $\phi$. The peak luminosity $\sim 2 \cdot 10^{31}$ $\mathrm{cm}^{-2} \mathrm{~s}^{-1}$ is limited while by a positrons injection rate and it will gain by a factor of ten after upgrade of the injection facility.

The relative difference of the luminosities determined with two processes versus energy is presented in Fig. 1, where only statistical errors are shown (SCAN 2011). The horizontal line is a fit for this ratio and in average it is about $0.2 \pm 0.3 \%$. The main sources which contribute to systematic 


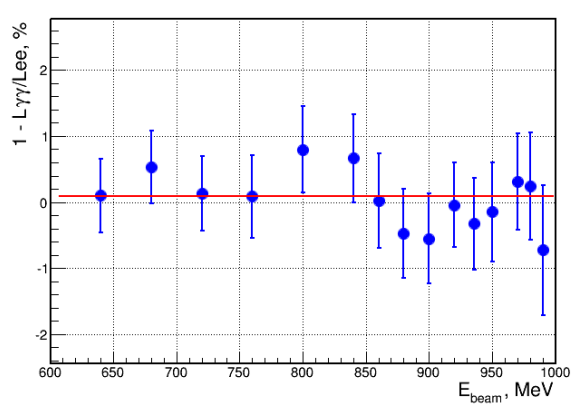

Figure 1. The ratio of the relative difference of the luminosities vs beam energy (scan 2011). Full circles - scan up, triangles - scan down. Line - fit: 0.2 $\pm 0.3 \%$

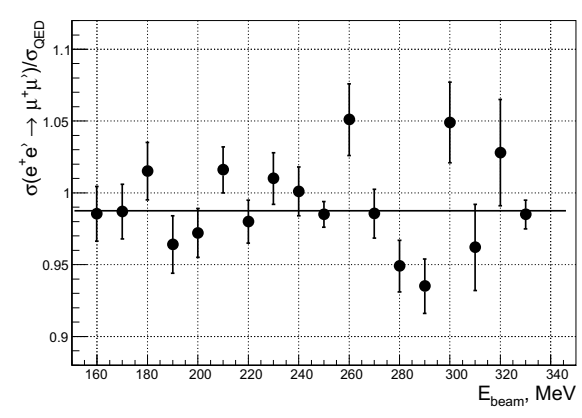

Figure 2. Result of the measurement of muon pair production in comparison with the QED prediction. Horizontal line - fit: $0.995 \pm 0.005$

error are: contribution due to the different angular resolutions for Bhabha events and $\gamma \gamma$ is estimated as $\sim 0.8 \%$; correction, which takes into account inclination of the beam axis with respect to detector is about $\sim 0.4 \%$; DC z-coordinate calibration contributes about $0.3 \%$; radiative corrections are calculated according to [9] with the accuracy about $0.2 \%$. Presently we estimate the current luminosity systematic accuracy as $\sim 1 \%$ for energies higher than $1 \mathrm{GeV}$.

\section{Processes with multihadrons in final states}

One of the main goal of the CMD-3 experiment is to reduce a systematic uncertainty of the cross section of two pion production to $0.3-0.4 \%$. The $\pi^{+} \pi^{-}$events are separated either using the particles momentum or their energy deposition in EM-calorimeter. Two ways of event separation will provide cross-check and is expected allow to keep the systematic error under control. The first energy scan below $1 \mathrm{GeV}$ was performed in 2013. The collected statistics is a few times higher than we had in the previous CMD-2 measurements and it is at the level of ISR statistics accumulated by the BaBar and KLOE. The process $e^{+} e^{-} \rightarrow \mu^{+} \mu^{-}$is very importance since it provides overall systematic test of the event separation accuracy. Preliminary results for the cross section $\sigma\left(e^{+} e^{-} \rightarrow \mu^{+} \mu^{-}\right)$are shown in Fig. 2 with respect to the QED prediction. Horizontal line is a fit for the double ratio $\sigma_{\mu \mu}^{\text {exp }} / \sigma_{\mu \mu}^{Q E D} / \sigma_{e e}^{\text {exp }} / \sigma_{e e}^{Q E D}$ which was found to be $0.995 \pm 0.005$. At the moment this result demonstrates our potential power of the event separation procedure. Study of different systematic uncertaintices is ongoing.

\subsection{Study of the processes $e^{+} e^{-} \rightarrow K_{S} K_{L}$ and $e^{+} e^{-} \rightarrow K^{+} K^{-}$.}

It is known the CMD-2 and BaBar results for cross sections at the $\phi$-peak region disagree at the level $\sim 4 \%$ for charged channel, so a new measurement are required. The $e^{+} e^{-} \rightarrow K_{S}^{0} K_{L}^{0}$ and $e^{+} e^{-} \rightarrow K^{+} K^{-}$ cross sections were measured in the c.m. energy range $1.004-1.060 \mathrm{GeV}$ at 25 energy points. The neutral mode detection is based on the search of two central tracks with common vertex in DC from the $K_{S}^{0} \rightarrow \pi^{+} \pi^{-}$decay. The number of events is defined by the fit of two pions invariant mass distribution [10].

The registration of the charged mode is based on the search of two central collinear tracks of kaons with defined momentum in DC. Each track should has ionization losses significantly larger than mip due to relatively small velocity of kaons under study. After these requirements the level of remaining 
background is less than $0.5 \%$. The detection efficiency of each kaon was determined with data and as well as with $\mathrm{MC}$ and deliver a deviation less $1.5 \%$.

The obtained cross sections for the neutral (published) and charged mode (preliminary) are presented in Fig. 3 and Fig. 4, correspondingly. The measured cross section is approximated according to VDM model as a sum of $\phi, \omega, \rho$-like amplitudes and their excitations. The interference of nonresonant amplitudes with the amplitude of $\phi$ meson scales in ten times and shown too at the bottom of graphs. The neutral and charged channels were approximated simultaneously, as a result the following values of the $\phi$ meson parameters have been obtained: $m_{\phi}=1019.464 \pm 0.060 \mathrm{MeV} / \mathrm{c}^{2}$, $\Gamma_{\phi}=4.240 \pm 0.017 \mathrm{MeV}, \frac{B_{\phi \rightarrow K^{+} K^{-}}}{B_{\phi \rightarrow K_{S}^{0} K_{L}^{0}}}=1.573 \pm 0.06$. The obtained parameters have accuracy comparable or better than it was obtained in previous experiments.

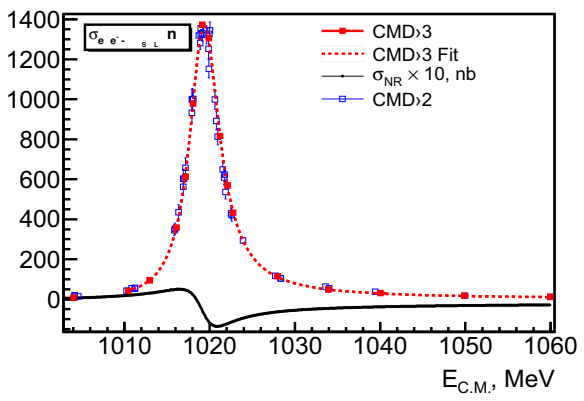

Figure 3. The cross section of the process $e^{+} e^{-} \rightarrow$ $K_{L} K_{S}$ around $\phi$-meson energy region. CMD-2, CMD-3 and BaBar data are presented.

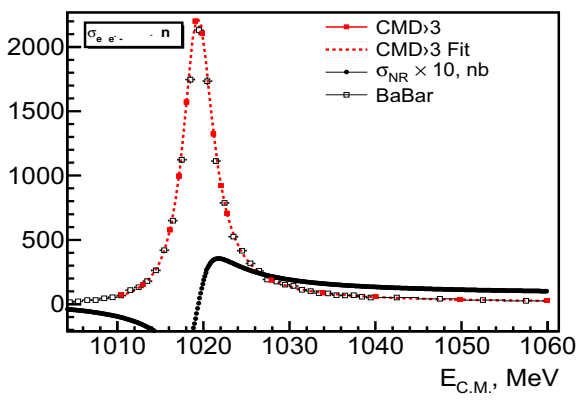

Figure 4. The cross section of the process $e^{+} e^{-} \rightarrow$ $K^{+} K^{-}$around $\phi$-meson energy region. CMD-2, CMD-3 and BaBar data are presented.

\subsection{Study of the process $e^{+} e^{-} \rightarrow K^{+} K^{-} \pi^{+} \pi^{-}$}

The cross section measurement of the process $e^{+} e^{-} \rightarrow K^{+} K^{-} \pi^{+} \pi^{-}$is based on the integrated luminosity of $22 \mathrm{pb}^{-1}$ in the c.m. energy range from 1.5 to $2.0 \mathrm{GeV}$ and early was measured by the BaBar via ISR. Nevertheless the direct measurements are very important, since some contributes to $a_{\mu}$ are based on isospin relations of various $K \bar{K}+n \pi$ final states. Any uncertainty of this approach will be crucial for the $a_{\mu}$ accuracy in the future calculations.

The signal events should have three or four tracks in the DC coming from interaction region and obey to the energy-momentum conservation. Two tracks corresponding to kaons should have specific ionization losses $\mathrm{dE} / \mathrm{dx}$ in DC. Fig. 5 shows the difference between measured total energy and c.m. energy $\Delta E_{4}=E_{t o t}-E_{c . m}$. vs the total momentum for all events with four tracks. The signal events are located near in origin of coordinates. The cluster of events with a zero total momentum but shifted up along the vertical axis, corresponds to $\pi^{+} \pi^{-} \pi^{+} \pi^{-}$events.

The similar procedure was used to select signal events with the three-track in DC. As a result, $\sim 13300$ four-track events and $\sim 16000$ three-track events were selected. To calculate a detection efficiency, the $K^{+} K^{-} \pi^{+} \pi^{-}$events were simulated with a primary generator using the GEANT4 package and then reconstructed with the same software as experimental data.

The cross section as a function of energy shown in Fig. 6, and well agrees with the previous BaBar measurement [11] presented by open circles. Systematic error was studied in detail and currently is 


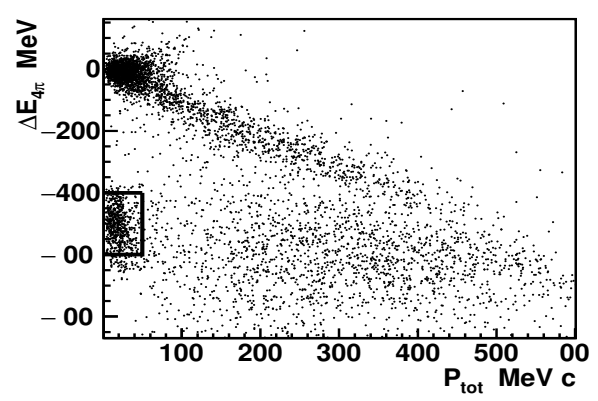

Figure 5. The difference between the total energy and c.m. energy $\left(\Delta E_{4}\right)$ versus the total momentum for the four-track events. The upper cluster of dots represents $\pi^{+} \pi^{-} \pi^{+} \pi^{-}$while the lower one $K^{+} K^{-} \pi^{+} \pi^{-}$events.

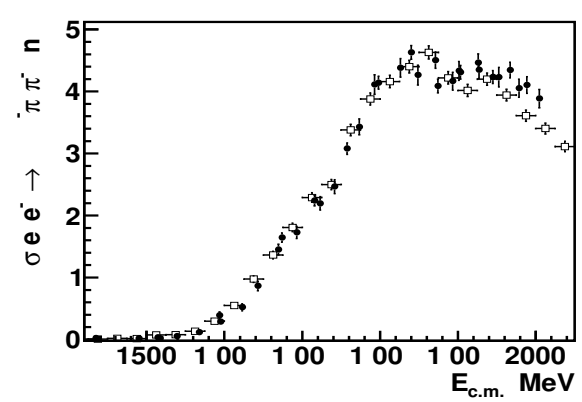

Figure 6. Dots - the $e^{+} e^{-} \rightarrow K^{+} K^{-} \pi^{+} \pi^{-}$cross section measured with the CMD-3. The BaBar results are shown by open circles.

estimated as $6 \%$ and mainly due to model dependence of the detection efficiency. More detail analyses can be found in publication [12].

\subsection{Six pion production}

Production of the six charged pions in $e^{+} e^{-}$annihilation was studied at DM2 [13] and BaBar [14]. The DM2 experiment observed a "dip" in the cross section of the process $e^{+} e^{-} \rightarrow 3\left(\pi^{+} \pi^{-}\right)$near $1.9 \mathrm{GeV}$, confirmed later by the BaBar. The origin of the "dip" remains unclear, but the most popular explanation is based on a hypothesis of a presence of the under-threshold $(p \bar{p})$ resonance discussed in many theoretical papers [15].

The analysis is based on $22 \mathrm{pb}^{-1}$ of the integrated luminosity collected in the c.m. energy range from 1.5 to $2 \mathrm{GeV}$. Candidates for the process under study are required to have five or six tracks in DC. For six- or five-track candidates the total energy and total momentum are calculated, assuming all tracks to be pions. To estimate the background MC simulation of the major processes $2\left(\pi^{+} \pi^{-} \pi^{0}\right)$ and $2\left(\pi^{+} \pi^{-}\right) \pi^{0}$ was performed and was found to be smaller than $1 \%$. The analysis in detail for this channel can be found in [16].

To measure the cross section of the process $e^{+} e^{-} \rightarrow 2\left(\pi^{+} \pi^{-} \pi^{0}\right)$ the sample of events with the four charged and two neutral pions were selected. To select neutral pions the spectrum of invariant mass of all two photon combinations was studied inside energy gap from $60<m_{\gamma \gamma}<200 \mathrm{MeV} / c^{2}$ and combination with the nearest to the pion mass is chosen. The number of events under study at each energy point was determined by fit of the distributions shown in Fig. 7 with a sum of three Gaussian functions for signal events and quadratic polynomial for background. The cross section calculates according to the number of determine events and takes into account RC and detection efficiency. The preliminary results for the cross section are presented in Fig. 8. The analysis of the data is in progress.

\subsection{Cross sections measurement of the $e^{+} e^{-} \rightarrow \eta \pi^{+} \pi^{-}$and $e^{+} e^{-} \rightarrow \omega \pi^{+} \pi^{-}$processes}

The process $e^{+} e^{-} \rightarrow \eta \pi^{+} \pi^{-}$was studied in two decay modes of $\eta: 2 \gamma$ and $\pi^{+} \pi^{-} \pi^{0}$. The signal events should have two tracks and at least two photons in EM-calorimeter. The shape of two photons invariant mass distribution was fix from MC and used to determine the number of the $\eta \pi^{+} \pi^{-}$events at 


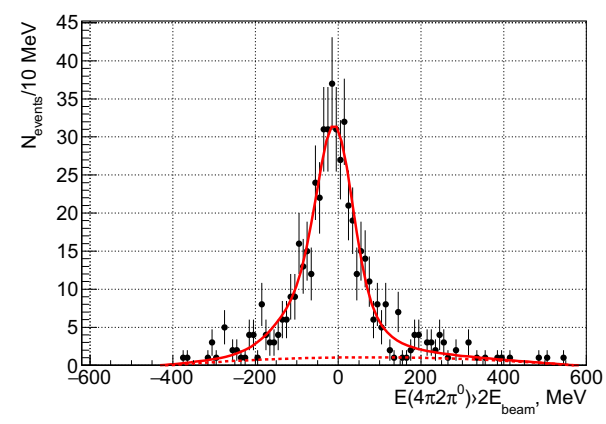

Figure 7. The number of events vs the total energy of the system $2\left(\pi^{+} \pi^{-} \pi^{0}\right)$. Signsl- fit with a sum of three Gaussian functions, background - fit with quadratic polynomial.

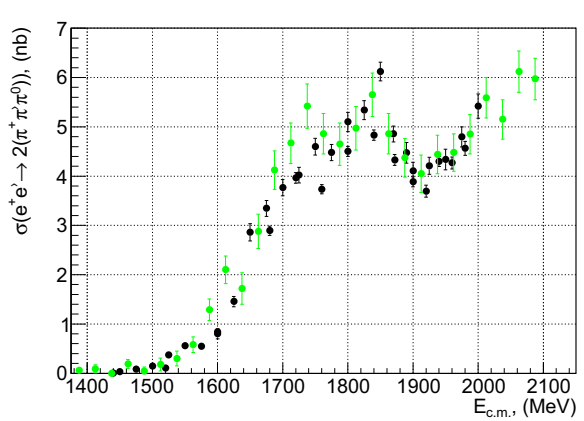

Figure 8. . Cross section of the process $e^{+} e^{-} \rightarrow$ $2\left(\pi^{+} \pi^{-} \pi^{0}\right)$ vs energy. Black points - CMD3, green BaBar.

each energy point. The result of such fit at $1500 \mathrm{MeV}$ is shown in Fig. 9. The preliminary results for the Born cross section are presented in Fig. 10. The systematic uncertainty for this process is about $5.2 \%$ and mainly due to uncertainty of detection efficiency, which depends on angular distribution of the final particles, i.e. depends on the intermediate states (theoretical models) through this process goes.

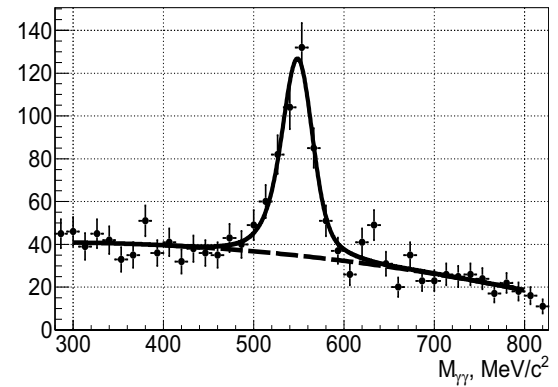

Figure 9. Two photons invariant mass at the energy of $1500 \mathrm{MeV}$.

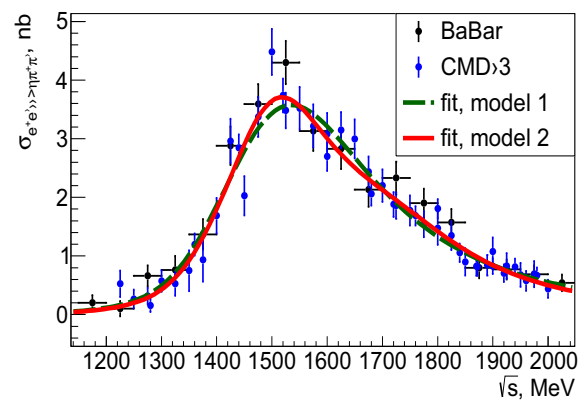

Figure 10. The $e^{+} e^{-} \rightarrow \eta \pi^{+} \pi^{-}$Born cross section measured in the $\eta \rightarrow \gamma \gamma$ channel. The results are presented together with BaBar data.

These two processes were studied too when $\eta$ and $\omega$ decay to the three pions: $\pi^{+} \pi^{-} \pi^{0}$. To determined the signal events the shape of the $\pi^{+} \pi^{-} \pi^{0}$ invariant mass distribution has been studied using Monte Carlo simulation and was used to count the number of the signal events. The preliminary results for the Born cross sections are consistent with other experiments. The current systematic uncertainty for these channels we estimated as $15 \%$ and mainly driven by the difference between angular distributions of charged particles in simulation and experiment. The analysis of systematic errors is ongoing. 


\subsection{Study of the processes $e^{+} e^{-} \rightarrow K^{+} K^{-} \eta$ and $e^{+} e^{-} \rightarrow K^{+} K^{-} \omega$}

The analysis of the process $e^{+} e^{-} \rightarrow K^{+} K^{-} \eta$ was based on $19 \mathrm{pb}^{-1}$ of an integrated luminosity collected by the CMD-3 detector in 2011-2012 in the $E_{\text {c.m. }}$. range from 1.59 to $2.01 \mathrm{GeV}$. On the base of these statistics we observed the contribution of $\phi(1020) \eta$ intermediate state only. Candidates for the events of the signal process were required to have two, three or four tracks in the DC, coming out of the beams intersection point. The kaon/pion separation was performed with the use of $f_{K / \pi}(p, d E / d x)$ functions [12], representing the probability density for charged kaon/pion with the momentum $p$ to produce the energy losses $d E / d x$ in the DC. We considered $\eta$-meson as a recoil particle, which allowed us to avoid the loss of statistics due to the selection of the specific $\eta$ decay mode. But such an inclusive approach lead to the complication of the signal/background separation. Therefore the major background processes were studied and were found to be $e^{+} e^{-} \rightarrow K^{+} K^{-} \omega(782), e^{+} e^{-} \rightarrow K^{+} K^{-} \pi^{+} \pi^{-}$, $e^{+} e^{-} \rightarrow \phi f_{0}(500), K^{* \pm}(892) K^{\mp} \pi^{0} \rightarrow K^{+} K^{-} \pi^{0} \pi^{0}, e^{+} e^{-} \rightarrow 2 \pi^{+} 2 \pi^{-} 2 \pi^{0}$. We perform the signal/background separation and $1296 \pm 43$ signal events were extracted. The resulting $e^{+} e^{-} \rightarrow \phi(1020) \eta$ cross section is shown in the Fig. 11 along with the BaBar results. The overall systematic uncertainty of the cross section measurement was estimated to be $6 \%$. Via the cross section approximation the $\phi(1680)$-meson parameters have been determined.

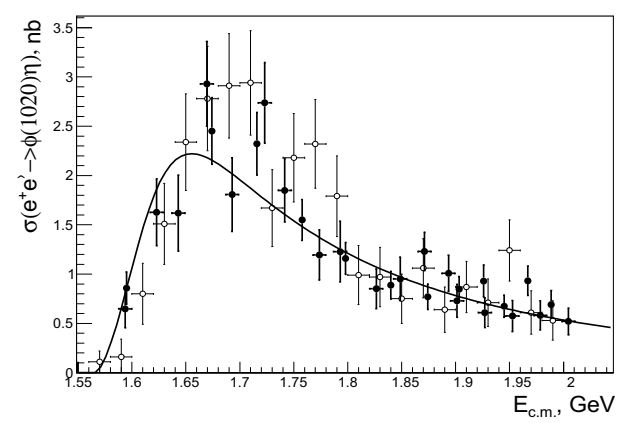

Figure 11. The BaBar (open circles) and CMD3 (filled circles) results for the measurement of $e^{+} e^{-} \rightarrow \phi(1020) \eta$ cross section in the range from 1.59 to $2.01 \mathrm{GeV}$. The approximation of CMD-3 data together with the $\mathrm{BaBar}$ data in the range from 2.3 to $3.46 \mathrm{GeV}$ is also shown.

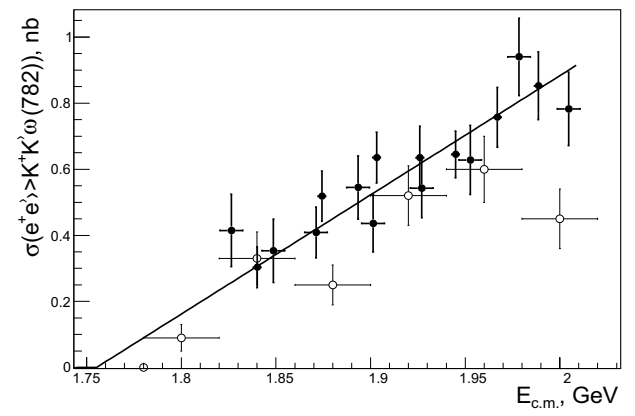

Figure 12. The BaBar (open circles) and CMD3 (filled circles) results for the measurement of $e^{+} e^{-} \rightarrow K^{+} K^{-} \omega(782)$ cross section in the range from 1.8 to $2.01 \mathrm{GeV}$. The approximation of CMD-3 data is also shown.

The analysis of the process $e^{+} e^{-} \rightarrow K^{+} K^{-} \omega(782)$ was based on $12 \mathrm{pb}^{-1}$ of the integrated luminosity collected by the CMD-3 detector in 2011-2012 in the $E_{\text {c.m. }}$. range from 1.8 to $2.01 \mathrm{GeV}$. Candidates for the events of the signal process were required to have three or four tracks in the DC, flying out of the area of the beams intersection. The kaon/pion separation was performed in the same way, as in the $e^{+} e^{-} \rightarrow K^{+} K^{-} \eta$ process analysis. We studied the process in the $\omega(782) \rightarrow \pi^{+} \pi^{-} \pi^{0}$ decay mode, considering $\pi^{0}$ as a recoil particle. The major background processes were found to be $e^{+} e^{-} \rightarrow K^{+} K^{-} \eta$ and $e^{+} e^{-} \rightarrow K^{+} K^{-} \pi^{+} \pi^{-}$, but their contribution was almost completely suppressed by the cuts on the invariant and missing masses of kaons and pions. After background suppression we performed a direct subtraction of the estimated number of background events and in total in the experiment we obtained $886 \pm 30$ signal events. The resulting $e^{+} e^{-} \rightarrow K^{+} K^{-} \omega(782)$ cross section is shown in the Fig. 12 along with the BaBar results. The overall systematic uncertainty of the cross section measurement was estimated to be $6 \%$. 


\section{Summary and conclusion}

CMD-3 detector will operate with a goal to get $\sim 1 \mathrm{fb}^{-1}$ in 5-10 years and provides the new precise results on the hadron production. The current integrated luminosity was measured using two well known QED processes $e^{+} e^{-} \rightarrow e^{+} e^{-}, \gamma \gamma$ and systematic accuracy is estimated as $1 \%$. Two type of the first level triggers «CHARGED» and «NEUTRAL» deliver the independent information that allowed to determine the detection efficiencies and to estimate their uncertainties. Data analysis is in progress, the already collected data sample provides the same or better statistical precision for the hadronic cross sections than in previous experiments were achieved.

\section{Acknowledgements}

The authors are grateful to A.I. Milstein for help with a theoretical interpretation and development of the models. We thank the VEPP-2000 team for excellent machine operation.

This work is supported in part by the Russian Education and Science Ministry, by FEDERAL TARGET PROGRAM Scientific and scientific-pedagogical personnel of innovative Russia in 20092013, by agreement 14.B37.21.07777, by the Russian Fund for the Basic Research grants.

Part of this work related to the photon reconstruction algorithm in the electromagnetic calorimeter is supported by the Russian Science Foundation (project No. 14-50-00080).

RFBR 10-02-00695-a, RFBR 10-02-00253-a, RFBR 11-02-00328-a, RFBR 11-02-00112-a, RFBR 12-02-31501-mol-a, RFBR 12-02-31499-mol-a, RFBR 12-02-31498-mol-a, RFBR 12-0201032-a, RFBR 13-02-00215-a.

\section{References}

[1] I. Koop et al., Nucl.Phys.B, Proc.Suppl. 181, 371 (2008)

[2] M. Davier et al., Eur. Phys. J. C 31, 503 (2003)

K. Hagiwara et al., J. Phys. G 38, 085003 (2011)

[3] G. Grawford et al., Nucl. Instr. Meth. A 345, 429 (1994)

[4] B. I. Khazin et al., Nucl.Phys.B, Proc. Suppl. 181-182, 376 (2008)

[5] V. M. Aulchenko et al, 2015 JINST 10 P10006 (2015)

[6] E. V. Abakumova et al., Phys. Rev. Lett. 110, 140402 (2013)

[7] E. A. Kuraev and V. S. Fadin, Yad. Fiz., vol.3 41, 733 (1984)

[8] S. I. Eidelman et al., Eur. Phys. J. C 71, 1597 (2011)

[9] A. B. Arbuzov et al. Eur. Phys. J. C 46, 689 (2006)

S. Actis et al., Eur. Phys. J. C 66, 585 (2010)

[10] E. A. Kozyrev et al., Phys. Lett. B 760, 314 (2016)

[11] B. Aubert et al. (BaBar Collaboration), Phys. Rev. D 76, 012008 (2007)

J. P . Lees et al. (BaBar Collaboration), Phys. Rev. D 86, 012008 (2012)

[12] D. N. Shemyakin et al., Phys.Lett. B 756, 153-160 (2016)

[13] R. Baldini et al., reported at the "Fenice" Workshop, Frascati (1988);

A. B. Clegg and A. Donnachie, Z. Phys. C 45, 677 (1990);

M. R. Whalley, J. Phys. G 29, A1 (2003)

[14] B. Aubert et al., (BaBar Collaboration), Phys. Rev. D 73, 052003 (2006)

[15] A. Sibirtsev and J. Haidenbauer, Phys. Rev. D 71, 054010 (2005)

A. Antonelli et al. (FENICE Collaboration), Phys. Lett. B 365, 427 (1989)

[16] R. R. Akhmetshin et al., Phys.Lett. B 723,82 (2013) 